\title{
Classical Model Validation for Control Design Purposes
}

\author{
Lennart $\mathrm{Ljung}^{+}$and Lei $\mathrm{Guo}^{++}$ \\ December 29, 1995
}

\begin{abstract}
Model Validation is at the heart of the System Identification process. Recently, much renewed interest has been expressed in so called "identification for control". This means that the design variables associated with the identification process are tailored to achieve models that are well suited for control design purposes. A separate, but closely related issue is to devise validation tests that give information about the model's quality and suitability for control design. This paper shows and discusses how a basic and classical residual test gives such information.
\end{abstract}

+ Department of Electrical Engineering, Linköping University, Linköping, S-58183, Sweden. Email: Ljung@isy.liu.se, Fax: (+46)13 282622.

++ Institute of Systems Science, Chinese Academy of Sciences, Beijing, 100080, P.R.China. Email: Lguo@iss03.iss.ac.cn, Fax: (86-10)2587343. 


\section{Introduction}

"Identification for Control" has since long been of main concern in the system identification literature. The identified model always has some intended application, and if it is control design, the model quality issues must be focused on that.

Model quality has two sides. One is that several design variables in the identification process affect the model properties in different ways, and we need to understand these influences. There is a very active, recent and renewed interest in various way to adapt the identification process to control design. See, e.g.,[3], [4], [17], [7].

Model validation is the other side of model quality. It has always played a major role in System Identification, as a basic instrument for model structure selection and as the last "quality control" station before a model is delivered to the user [11], [20]. Methods for robust control design have pointed to the need for reliable model error bounds, for linear models preferably described as bounds on the frequency functions. A large number of approaches have been developed for this. See, e.g., [9],[10],[6],[5],[21]. For recent work on model validation in a worst-case context see [15] and [19]. Many of the contributions use deterministic frameworks to describe the noise and disturbances appearing in the system in order to avoid probabilistic, "soft", bounds. Approaches like "unknown-but-bounded" noises (the disturbances are assumed to be bounded, but no other assumptions are invoked), see e.g. [18], lead to set-membership procedures, which determine all models that are consistent with the noise bound given, see, e.g. [1], [14], [22].

In this contribution we shall review some of the basic issues in this process. In Section 2 guidelines for selecting appropriate design variables for the identification process are briefly reviewed. Section 3 deals with the underlying principles of model validation in general terms, while Section 4 reviews a recent result on how a classical validation test can be translated to the frequency domain. In Section 5 we discuss some issues around model validation for FIR models and unknown-but-bounded disturbances. Section 6 deals with the question of performing the validation directly in the frequency domain. 


\section{Some Notations}

We shall use the following notation. The input will be denoted by $u(t)$ and the output by $y(t)$. The data record thus is

$$
Z^{N}=\{y(1), u(1), \ldots, y(N), u(N)\}
$$

The input sequence $\{u(t), t=1, \ldots, N\}$ will throughout this paper be considered as a deterministic sequence, unless otherwise stated. We denote its periodogram by

$$
\left|U_{N}(\omega)\right|^{2}=\frac{1}{N}\left|\sum_{t=1}^{N} u(t) e^{-i \omega t}\right|^{2}
$$

The given model $\hat{G}$ will be assumed to be linear, and a function of the shift operator $q$ in the usual way: $\hat{G}(q)$. The simulated output will thus be

$$
\hat{y}(t)=\hat{G}(q) u(t)
$$

It may be that the model contains a noise assumption, typically in the form of an additive noise or disturbance $v(t)$ with certain properties. It would then be assumed that the actual output is generated as

$$
y_{m}(t)=\hat{G}(q) u(t)+v(t)
$$

The model could contain some "prejudice" about the properties of $v(t)$, but this is not at all essential to our discussion. A typical, conventional assumption would be that $v(t)$ is generated from a white noise source through a linear filter:

$$
v(t)=\hat{H}(q) e(t)
$$

Most of the model validation tests are based on simply the difference between the simulated and measured output:

$$
\varepsilon(t)=y(t)-\hat{y}(t)=y(t)-\hat{G}(q) u(t)
$$

For added generality, we shall consider possibly prefiltered model errors:

$$
\varepsilon(t)=L(q)[y(t)-\hat{y}(t)]=L(q)[y(t)-\hat{G}(q) u(t)]
$$

For example, if the model comes with a noise model (5), then a common choice of prefilter is $L(q)=\hat{H}^{-1}(q)$, since this would make $\varepsilon(t)$ equal to the model's prediction errors. This choice of prefilter is however not at all essential to our discussion.

In any case we shall call $\varepsilon(t)$ the Model Residuals ("model leftovers"). 


\section{Design of the System Identification Process in the Frequency Domain}

There is a recent interest in system identification directly from frequency domain data, as described in e.g., [16], [12]. Most "classical" approaches otherwise take place in the time domain, both when it comes to the models, algorithms and model validation techniques. On the other hand, the model properties for control design are most of the time best expressed and evaluated in the frequency domain.

Most links between the time domain (prediction error) identification methods and the model's frequency domain properties follow from the property

$$
\begin{array}{cl}
\text { Model: } & y(t)=G(q, \theta) u(t)+H(q, \theta) e(t) \\
\text { Criterion: } & V_{N}\left(\theta, Z^{N}\right)= \\
& \frac{1}{2 \pi} \int_{-\pi}^{\pi} \frac{1}{2}\left|\hat{\hat{G}}_{N}\left(e^{-i \omega}\right)-G\left(e^{i \omega}, \theta\right)\right|^{2} Q_{N}(\omega, \theta) d \omega \\
\text { where } & \hat{\hat{G}}_{N}\left(e^{i \omega}\right)=\frac{Y_{N}(\omega)}{U_{N}(\omega)} \\
\text { and } & Q_{N}(\omega)=\frac{\left|U_{N}(\omega)\right|^{2}}{\left|H\left(e^{i \omega}, \theta\right)\right|^{2}}
\end{array}
$$

(see, e.g., eq (7.25) in [11].) Here $U_{N}(\omega)$ is the discrete time Fourier transform of the input

$$
U_{N}(\omega)=\frac{1}{\sqrt{N}} \sum_{t=1}^{N} u(t) e^{-i \omega t}
$$

The resulting model parameters are then given by

$$
\hat{\theta}_{N}=\arg \min V_{N}\left(\theta, Z^{N}\right)
$$

The design variables associated with the identification process are then primarily the experimental conditions (in open loop, the input spectrum) and the data prefilters (which, for SISO systems are equivalent to the noise model $H(q, \theta)$, and closely related to the predictors' prediction horizon; see [11].).

An identification task to optimize the model quality for a particular poleplacement control design can then be formulated as in eq (12.23) in [11]:

$$
\min \int_{-\pi}^{\pi} \mathrm{E}\left|\hat{G}\left(e^{i \omega}\right)-G_{0}\left(e^{i \omega}\right)\right|^{2} \frac{\left|R\left(e^{i \omega}\right)\right|^{2} \Phi_{r}(\omega)}{\left|G_{0}\left(e^{i \omega}\right)\right|^{2}\left|1+G_{0}\left(e^{i \omega}\right) F_{2}\left(e^{i \omega}\right)\right|^{2}} d \omega
$$


Here $\Phi_{r}$ is the spectrum of the reference signal and $R(q)$ is the desired closed loop system, while $F_{2}(q)$ is the feedback part of the (as yet unknown) regulator. (Consequently

$$
\frac{1}{1+G_{0}(q) F_{2}(q)}
$$

is the (unknown) sensitivity function.)

The minimization in (14) is to be carried out over all available identification design variables, including possible feedback controls. The solution is, according to Theorem 14.4 in [11] to perform an open loop experiment with input spectrum

$$
\Phi_{u}(\omega)=\mu_{1} \frac{\left|R\left(e^{i \omega}\right)\right| \Phi_{r}(\omega)}{\left|G_{0}\left(e^{i \omega}\right)\right|\left|1+G_{0}\left(e^{i \omega}\right) F_{2}\left(e^{i \omega}\right)\right|} \sqrt{\Phi_{r}(\omega) \Phi_{v}(\omega)}
$$

( $\Phi_{v}$ is the additive disturbance spectrum) and a prefilter=noise model

$$
\left|H\left(e^{i \omega}\right)\right|^{2}=\mu_{2} \frac{\Phi_{v}(\omega)}{\Phi_{u}(\omega)}
$$

A problem with this - as most of the time with optimal design - is that the solution depends on unknown quantities (like $G_{0}$ ). Several iterative schemes for the design have therefor been developed. See, among many references, [4], [17], [7].

However, in this contribution, the validation of the resulting model is our main concern, rather than the identification design. Our main result will actually concern the translation of the basic, classical validation test to the frequency domain, in much the same way as (8).

\section{Some Principles of Model Validation}

We place ourselves in the following situation. A model is given. Let it be denoted by $\hat{G}$ (more specific notation will follow later). We are also given a data set $Z^{N}$ consisting of measured input-output data from a system. We do not know, or do not care, how the model was estimated, or constructed or given. We might not even know if the data set was used to construct the model.

Our problem is to figure out if the model $\hat{G}$ is any good at describing the measured data, and perhaps also to give a statement how "far away" 
the model might be from a true description. We would like to approach this problem as naked as possible, and strip off common covers, such as "prior assumptions", "probabilistic frameworks", "worst case model properties" and the like. What are we then left with?

Well, a natural start is to consider the model's simulated response to the measured input signal. Let that simulated output be denoted by $\hat{y}$. We would then compare this model output with the actual measured output and contemplate how good the fit is. This is indeed common practice, and is perhaps the most useful, pragmatic way to gain confidence in (or reject) a model. This will be the starting point of our discussion.

In the end, all we really have to our disposal for model validation is the sequence of residuals, computed on "historic data". We can give different kind of statistics for these residuals, like the maximum absolute value, or the mean square value. The implication would be that there is some kind of "invariance principle" for the system, so that these statistics will be valid also in the future use of the model.

This is one part of the essence of model validation. The other part is to figure out if there is reason to believe that this "invariance" also will be valid if the character of the input is changed:

"Here is a model. On past input-output data it has produced model errors with certain statistics. If I change the input $u$, will then the errors be significantly different?"

To check the part of the residuals that might originate from the input the following statistics are frequently used (see e.g., [2].):

Let

$$
\varphi(t)=[u(t), u(t-1), \ldots, u(t-M+1)]^{T}
$$

and

$$
R_{N}=\frac{1}{N} \sum_{t=1}^{N} \varphi(t) \varphi(t)^{T}
$$

Now form the following scalar measure of the correlation between past inputs (i.e. the vector $\varphi$ ) and the residuals:

$$
\tilde{\xi}_{N}^{M}=\frac{1}{N}\left|\sum_{t=1}^{N} \varphi(t) \varepsilon(t)\right|_{R_{N}^{-1}}^{2}
$$


Note that this quantity also can be written as

$$
\tilde{\xi}_{N}^{M}=\hat{r}_{\varepsilon u}^{T} R_{N}^{-1} \hat{r}_{\varepsilon u}
$$

where

$$
\hat{r}_{\varepsilon u}=\left[\hat{r}_{\varepsilon u}(0), \ldots, \hat{r}_{\varepsilon u}(M-1)\right]^{T}
$$

with

$$
\hat{r}_{\varepsilon u}(\tau)=\frac{1}{\sqrt{N}} \sum_{t=1}^{N} \varepsilon(t) u(t-\tau)
$$

It is clear that the induction about the size of the model residuals from one data set to another is much more reasonable if the statistics $\tilde{\xi}_{N}^{M}$ has a small value.

To come up with measures of how well the model describes the true system, we shall assume that the data $Z^{N}$ have been generated by a "true system"

$$
y(t)=G_{0}(q) u(t)+v(t)
$$

and we define the discrepancy as

$$
\tilde{G}(q)=G_{0}(q)-\hat{G}(q)
$$

\section{Some Basic Limitations}

Model validation is really about model falsification. That is, we try to establish convincing evidence that a certain model cannot have produced the observed data. A model that "so far" has not been falsified can be seen - for the moment - as "validated".

It is also the case that the process of validation hinges upon prior knowledge or prior hypothesis of different kinds. This is unavoidable. A trivial way to realize that this must be the case is the time/frequency uncertainty principle: After having seen $N$ data points we know nothing about the model's frequency behavior with a resolution less than $2 \pi / N$ radians/sampling interval. The validation process must then be complemented either by priors on model order or on frequency function smoothness. The latter is related to the rate of decay of the impulse response.

With statistics like (19), we are only probing dynamics up to lag $M$. This means that we do not check the impulse response beyond this lag. Any statement about the model quality must thus hinge upon assumptions/knowledge about the tail of the impulse response. 


\section{Translating the Basic Model Validation Test to the Frequency Domain}

The question now is, what can be said about the model error $\tilde{G}$ based on the information in $Z^{N}$.

The procedure will be to form

$$
\varepsilon(t)=L(q)(y(t)-\hat{G}(q) u(t))
$$

and then $\tilde{\xi}_{N}^{M}$ as in (17)-(19). In these calculations replace $u(t)$ outside the interval $[1, N]$ by zero. Assume that $R_{N}>\delta I$. It is then shown in [13] that

$$
\begin{aligned}
& {\left[\frac{1}{2 \pi} \int_{-\pi}^{\pi}\left|\tilde{G}\left(e^{i \omega}\right)\right|^{2}\left|L\left(e^{i \omega}\right)\right|^{2}\left|U_{N}(\omega)\right|^{2} d \omega\right]^{1 / 2} \leq } \\
\leq & (1+\eta)\left[\frac{1}{N} \tilde{\xi}_{N}^{M}\right]^{1 / 2}+(1+\eta) x_{N}+(2+\eta) C_{u} \sum_{k=M}^{\infty}\left|\rho_{k}\right|
\end{aligned}
$$

Here

- $x_{N}=\left|\frac{1}{N} \sum_{t=1}^{N} \tilde{v}(t) \varphi(t)\right|_{R_{N}^{-1}}$

- $\tilde{v}(t)=L(q) v(t)$

- $\rho_{k}$ is the impulse response of $L(q) \tilde{G}(q)$

- $\left|U_{N}\right|^{2}$ is the periodogram (2).

- $\eta=\frac{C_{u} M}{\sqrt{N \delta}}$

- $C_{u}=\max _{1 \leq t \leq N}|u(t)|$.

If the input is tapered so that $u(t)=0$ for $t=N-M+1, \ldots N$, the number $\eta$ can be taken as zero.

Let us make a number of comments:

- The result is really just a statement about the relationship between the sequences $\tilde{v}(t)=L(q)\left[y(t)-G_{0}(q) u(t)\right]$, and $\varepsilon(t)=L(q)[y(t)-\hat{G}(q) u(t)]$ on the one hand and the given transfer functions $L(q), G_{0}(q), \hat{G}(q)$ together with the given sequences $u(t), y(t)$ on the other hand. There are as yet no stochastic assumptions whatsoever, and no requirement that the "model" $\hat{G}$ may or may not be constructed from the given data. 
- By the choice of prefilter $L(q)$ we can probe the size of the model error over arbitrarily small frequency intervals. However, by making this filter very narrow band, we will also typically increase the size of the impulse response tail. (Narrow band filters have slowly decaying impulse responses.)

- In practical use the often erratic periodogram $\left|U_{N}\right|$ can be replaced by smoothed variants.

- For the quantities on the right hand side, we note that $\tilde{\xi}_{N}^{M}$ is known by the user, as well as $\eta, N$ and $C_{u}$. The tail of the impulse response $\rho_{k}$ beyond lag $M$ is typically not known. It is an unavoidable term, since no such lag has been tested. The size of this term has to be dealt with by prior assumptions.

- The only essential unknown term is $x_{N}$. We shall call this "The correlation term". The size and the bounds on this term will relate to noise assumptions and we will deal with these in some detail in the two following sections.

The implications of this result under varying assumptions about the additive disturbance $v(t)$ are discussed in [13]. We shall here make some comments related to control design applications. We shall then concentrate on a nonprobabilistic framework.

\section{Example application: FIR models and unknown- but-bounded disturbances}

The term $x_{N}$ measures the correlation between the input $u$ and the filtered disturbance $\tilde{v}$. In a deterministic setting it is not so easy to formalize what we should mean by "uncorrelated disturbances". One could of course postulate that the disturbance sequence that we expect to enter the process is such that quantities like $x_{N}$ decay like $1 / N$ or $\log N / N$ or in any other way.

From [13] we also have the following results: 


\section{A simple bound}

Suppose that $\tilde{v}(t)$ is any sequence, and all that is known about it is an amplitude or an energy bound.

$$
x_{N} \leq\left[\frac{1}{N} \sum_{t=1}^{N} \tilde{v}^{2}(t)\right]^{1 / 2}
$$

A bound on disturbance power or amplitude will thus directly give a hard model error bound in (25).

\section{The Case of Periodic Input}

If the input is periodic with period $P$, then

$$
x_{N} \leq C_{N} \cdot \max _{\omega}|\tilde{V}(\omega)|
$$

where $\tilde{V}(\omega)$ is the discrete time Fourier transform of $\tilde{v}(t),(\operatorname{cf}(12))$ and

$$
C_{N}=\frac{1+\log (N / P+1)}{\sqrt{N}} \cdot \frac{\sqrt{M} P}{\sqrt{\delta}} \cdot C_{u}
$$

The lemma says that for periodic input and for noises with suitably smooth spectrum, the model error essentially decays like $O\left(\frac{1}{\sqrt{N}}\right)$. This is the same type of result that is obtained in the classical stochastic framework.

\section{Model Validation with FIR Models}

The result (25) can be used in a variety of ways. We will see in the next section how a given model can be probed using validation data and different prefilters.

Another illustration is as follows. Suppose that the data has been generated by a system

$$
y(t)=G_{0}(q) u(t)+v(t)
$$

where all that is known about $v(t)$ is that it is bounded:

$$
|v(t)| \leq C_{v}
$$


Suppose that the model $\hat{G}_{N}$ is estimated using $Z^{N}$ as an $M$ :th order FIR (finite impulse response) filter using the least squares method. Then by construction, $\tilde{\xi}_{N}^{M}$ is zero. By (25) and (26) we then have the following result:

$$
\begin{aligned}
& {\left[\frac{1}{2 \pi} \int_{-\pi}^{\pi}\left|G_{0}\left(e^{i \omega}\right)-\hat{G}_{N}\left(e^{i \omega}\right)\right|^{2}\left|U_{N}(\omega)\right|^{2} d \omega\right]^{1 / 2} \leq } \\
\leq & (1+\eta) C_{v}+(2+\eta) C_{u} \sum_{k=M}^{\infty}\left|\rho_{k}\right|
\end{aligned}
$$

where $\rho_{k}$ is the impulse response of $G_{0}(q)$. The variable $\eta$ was defined following eqn (25).

Under such very weak assumption about the disturbance, we cannot, with this result, come below a certain lower limit for the fit, no matter how large we choose $N$ and $M$. On the other hand, a good signal to noise ratio $\left(C_{v} /\left|U_{N}\right|\right.$ small) can give very good fits by making $M$ sufficiently large. (To keep $\eta$ small, $N$ must increase faster than $M^{2}$.)

It might also be noted that without further assumptions about $v$, we don't gain much ("certified") model quality by concentrating the fit to certain frequency bands: If the data is prefiltered before used for estimation, so that the power of $\left|U_{N}(\omega)\right|$ is concentrated to certain frequency bands, the right hand side of (31) does not decrease. This follows from the fact that the power of $v$, as measured in (26) could be concentrated to the same frequency bands as those selected by the prefilter.

\section{Model Validation in the Frequency Domain}

The traditional way of validating models is, as we have seen, figures like Fig 2, where the cross correlation between residuals and input (regressors) is plotted. From a control design point of view, it would be much more natural to rather look at the Fourier transform of this plot, i.e. the cross spectrum between input and residuals. That will tell not only the integrated size of the model error $\tilde{G}_{N}\left(e^{i \omega}\right)$, as in $\tilde{\xi}$ in (19), but also how it is distributed over frequencies. The usefulness of looking at this cross spectrum was pointed out by Kosut in [8], but has not been further elaborated. The idea is illustrated by the following example.

Example 6.1 The fourth order system

$$
y(t)-2.2 y(t-1)+2.42 y(t-2)-1.87 y(t-3)+0.7225 y(t-1)
$$




$$
=u(t-1)+0.5 u(t-2)
$$

was simulated with a binary, white input and negligible additive noise. Two second order ARX models were estimated, one based on the original data set and one based on the data filtered through a (5th order, Butterworth) low pass filter with cut-off frequency $0.2 \pi$. The amplitude Bode plots of these two models, together with the true system are shown in Figure 1.

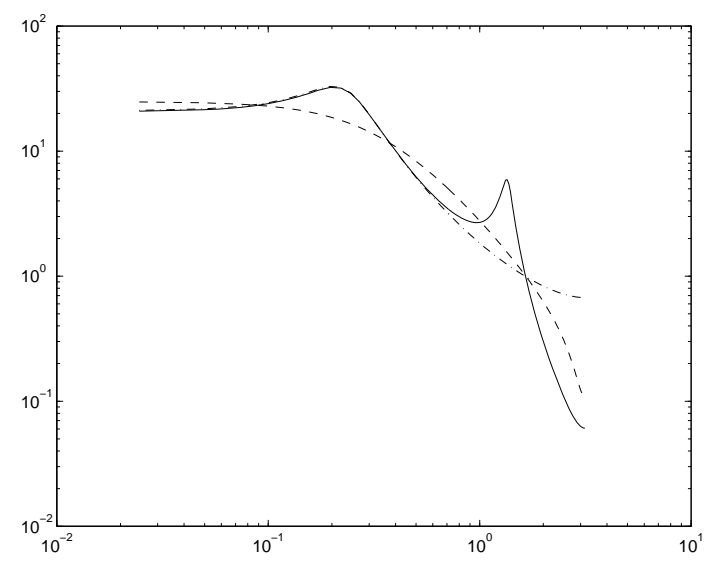

Figure 1: Bode amplitude plots. Solid: True system. Dashed: Model based on unfiltered data. Dash-dotted: Model based on filtered data.

The standard cross-correlation residual tests (both performed for the original, unfiltered data) are shown in figures 2 and 3. Applying the result (25) would give a large value of the test quantity $\tilde{\xi}$ and - correctly so - tell us that there is a significant discrepancy between both models and the true system, when evaluated over the whole frequency range (The periodogram $\left|U_{N}(\omega)\right|$ is flat). The test would not tell us the character of the discrepancy.

Estimating the transfer function from $u$ to $\varepsilon$ using spectral analysis, gives the results shown in Figures 4 and 5.

We see that these two figures give correct information about the reliability of the transfer function estimates. The information is consistent with Figure 1 (which of course will not be known to the user.)

To use the classical residual test, and result (25), supposing we were interested in the model fit over the frequency range $\left[\begin{array}{ll}0 & 0.2 \pi\end{array}\right]$, we should prefilter the residuals, so that $L(q)$ in (25) picks out the desired range. Residual plots for such prefiltered data are shown in Figures 6 and 7 . 

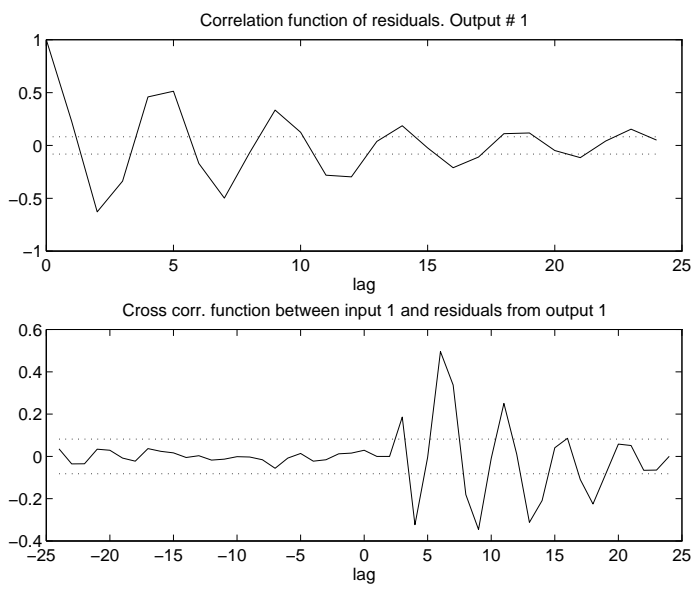

Figure 2: Residual test for the second order model estimated using the unfiltered data


Figure 3: Residual test for the second order model estimated using the filtered data. The test is performed on the original data 


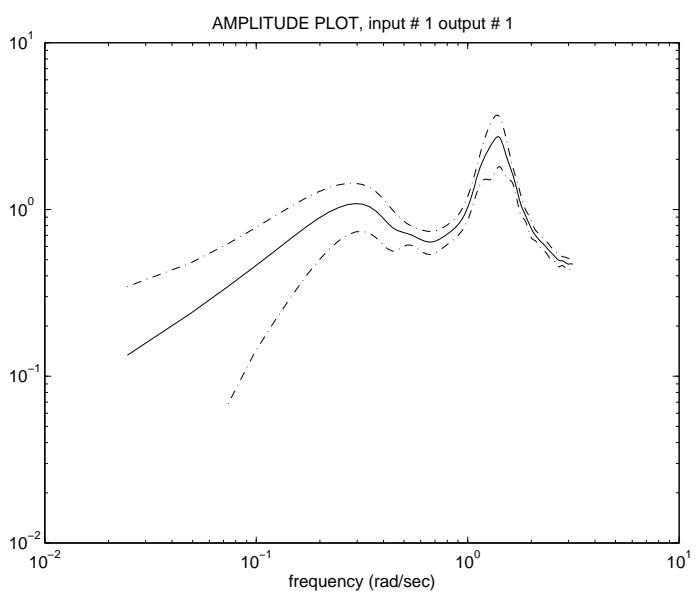

Figure 4: Amplitude plot with confidence interval corresponding to 3 standard deviations for the transfer function from input to residuals from the second order ARX model, estimated from unfiltered data.

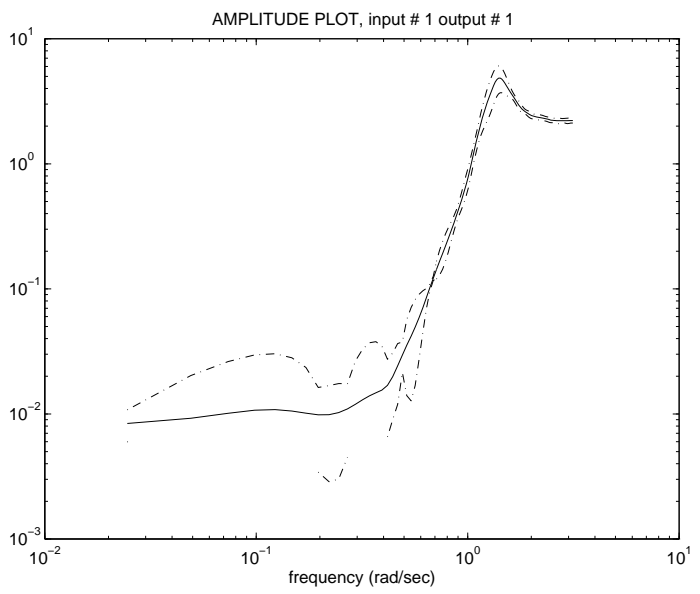

Figure 5: As Figure 4, but using residuals from the model obtained by filtered data. 

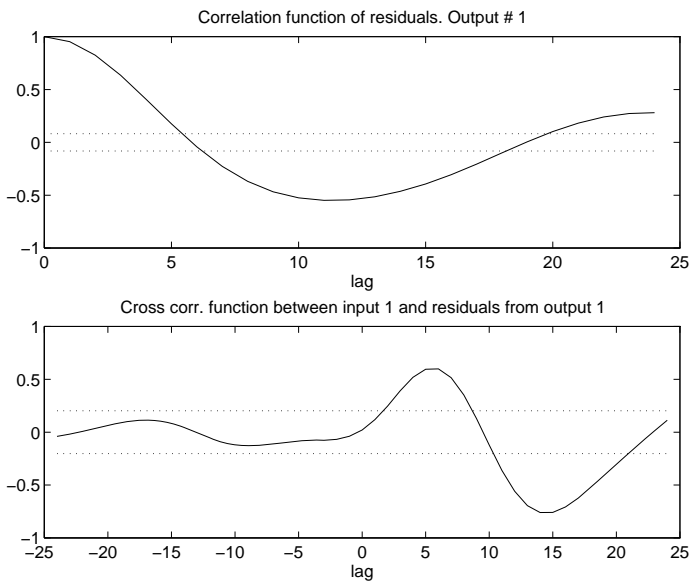

Figure 6: Residual test for the second order model estimated using the unfiltered data. Test performed on filtered data.
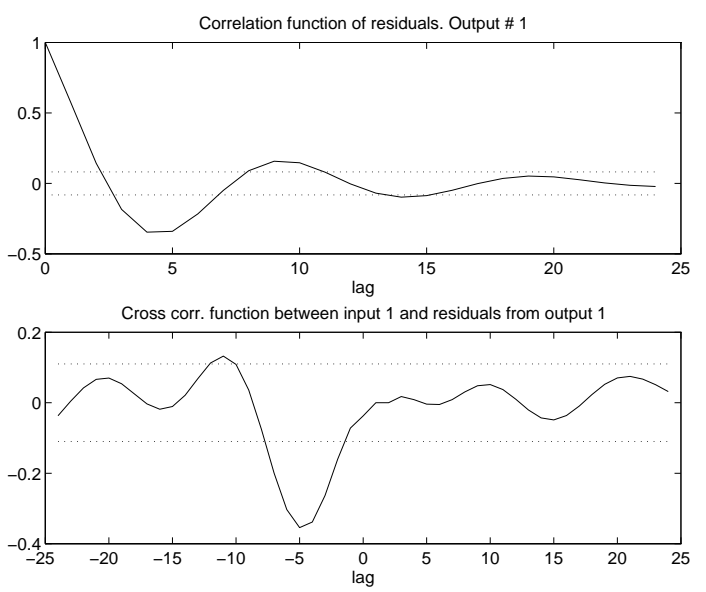

Figure 7: Residual test for the second order model estimated using the filtered data. Test performed on filtered data. 
Note that the measure $\tilde{\xi}$ only uses the correlation for positive lags. Consequently the "filtered model" in Figure 7 will pass the test, and - correctly so - tell us that the model fit over the frequency range of interest is good, according to (25).

\section{Conclusions}

The classical paradigm for model validation tells us that we should build more and more complex models (like increasing the model order) until a model is found that passes model validation tests, like

$$
\tilde{\xi}_{N}^{M} \leq C_{\xi}
$$

The "identification-for-control" movement has in a sense rejected this attitude, by stressing that control design might require simpler models than so, and that we can very well live with model errors that are statistically significant. It is just a matter of choosing the identification design variables so that these model errors fall into frequency regions that can be well handled by feedback. The challenge is then to evaluate the model errors without having access to a validated model.

Looking back at (25), we see a result that tells us the (weighted frequency domain) quality of a given model, regardless of whether it is "validated" or not. To use it we must invoke knowledge/assumptions about the nature of the disturbances (for $x_{N}$ ) and about the tail of the true system's impulse response. Such assumptions in one or another form are unavoidable.

We also see how the classical validation criterion (33) becomes natural in this context: It makes the two first terms of the right hand side of (25) balance each other under the typical probabilistic assumptions about $v$ (making $x_{N}$ behave like $1 / \sqrt{N}$ ). Note that this also covers the deterministic case $(27)$. In this light it is always natural to press the model to such a point that (33) holds. Then we have the best possible knowledge about its discrepancy from the true system. If the model is too complex to be used for control design, it can always to reduced: In that case we know exactly the difference between the validated model and the reduced one. That can be translated to a less conservative error bound on the reduced order model, used for the control design.

In fact, the process illustrated in Section 6, with spectral analysis of the transfer function from $u$ to the residuals is of the same nature. If we had done 
FIR modeling of the transfer function from $u$ to $\varepsilon$ the sum of the nominal model $\hat{G}$ and the one estimated using $\varepsilon$ would have constituted a validated model.

The bottom line seems to be that even for control oriented model validation there is no real escape from the classical paradigm: Obtain an unfalsified model: reduce it if necessary.

\section{References}

[1] J.R. Deller. Set membership identification in digital signal processing. IEEE ASSP Magazine, 4:4-20, 1989.

[2] N.R. Draper and H. Smith. Applied Regression Analysis, 2nd ed. Wiley, New York, 1981.

[3] M. Gevers and L. Ljung. Optimal experiment designs with respect to the intended model application. Automatica, 22:543-555, 1886.

[4] Michel Gevers. Towards a joint design of identification and control? In H L Trentelman and J C Willems, editors, Essays on control: Perspectives in the theory and its applications, ECC '93 Groningen, 1993.

[5] G.C. Goodwin, M. Gevers, and B. Ninness. Quantifying the error in estimated transfer functions with application to model order selection. IEEE Trans. Automatic Control, 37(7):913-929, 1992.

[6] G.C. Goodwin and M. Salgado. A stochastic embedding approach for quantifying uncertainty in estimation of restricted complexity models. Int. J. of Adaptive Control and Signal Processing, 3:333-356, 1989.

[7] H. Hjalmarsson, M. R. Gevers, F. De Bruyne, and J. Leblond. Identification for control: Closing the loop gives more accurate controllers. In Proceedings of the 33rd IEEE Conference on Decision and Control, pages 4150-4155, Lake Buena Vista, Florida, 1994.

[8] R.L. Kosut. Adaptive calibration: An approach to uncertainty modeling and on-line robust control design. volume 1 of Proc. 25th IEEE Conference on Decision an Control, pages 455-461, Athens, Greece, December 1986. 
[9] R.L. Kosut, G. C. Goodwin, and M. P. Polis (Eds). Special Issue on System Identification for Robust Control Design, IEEE Trans. Automatic Control, Vol 37. 1992.

[10] R.L. Kosut, M.K. Lau, and S.P. Boyd. Set-membership identification of systems with parametric and nonparametric uncertainty. IEEE Trans. Autom. Control, 37(7):929-942, 1992.

[11] L. Ljung. System Identification - Theory for the User. Prentice-Hall, Englewood Cliffs, N.J., 1987.

[12] L. Ljung. Building models from frequency domain data. In K.J. Åström, G.C.Goodwin, and P.R Kumar, editors, Adaptive Control, Filtering and Signal Processing, volume 74 of The IMA volumes in Mathematics and its Applications, pages 229-240. Springer-Verlag, New York, 1995.

[13] L.Ljung and L. Guo. The role of model validation for assessing the size of the unmodeled dynamics. Technical Report LiTH-ISY-I-1803, Submitted to IEEE Trans. Automatic Control, Dept. of Electrical Engineering, Linköping University, Sweden. Also available by ftp., 1995.

[14] M. Milanese and R. Tempo. Optimal algorithms for robust estimation and prediction. IEEE Trans. Automatic Control, AC-30:730-738, 1985.

[15] K. Poolla, P. P. Khargonekar, A. Tikku, J. Krause, and K.Nagpal. A time-domain approach to model validation. IEEE Trans. on Automatic Control, AC-39:951-059, 1994.

[16] J. Schoukens and R. Pintelon. Identification of Linear Systems: A Practical Guideline to Accurate Modeling. Pergamon Press, London (U.K.), 1991.

[17] R. Schrama. Control-oriented approximate closed-loop identification via fractional representations. In Proc. American Control Confernce, pages 719-720, Boston, MA, 1991.

[18] F.C. Schweppe. Uncertain Dynamical Systems. Prentice-Hall, Englewood Cliffs, 1973.

[19] R.S. Smith and J.C Doyle. Model invalidation: A connection between robust control and identification. IEEE Trans. Automatic Control, 37:942952, July 1992. 
[20] T. Söderström and P. Stoica. System Identification. Prentice-Hall International, Hemel Hempstead, Hertfordshire, 1989.

[21] B. Wahlberg and L. Ljung. Hard frequency-domain model error bounds from least-squares like identification techniques. IEEE Trans. on Automatic Control, pages 900-912, 1992.

[22] E. Walter and H. Piet-Lahanier. Exact and recursive description of the feasible parameter set for bounded error models. In Proc 26 IEEE Conf. on Decision and Control, pages 1921-1922, Los Angeles, 1987.

ljung/papers/modval/mmos.tex 